\title{
ENANTIOMERIC LC SEPARATION OF METOLAZONE ON AMYLOSE BASED SORBENT
}

\author{
VIPUL. P. RANE, KIRAN. R. PATIL, RAVINDRA. D.YEOLE, DEVANAND .B. SHINDE* \\ Department of Chemical Technology, Dr. Babasaheb Ambedkar Marathwada University, \\ Aurangabad- 431004 (MS), India
}

(Received: May 2, 2012 - Accepted: October 6, 2012)

\begin{abstract}
A simple, rapid and robust LC method was developed and validated for the enantiomeric separation of metolazone. The enantiomers of metolazone were resolved on a Chiralpak AD-H (amylose based stationary phase) column using a mobile phase consisting of Hexane: 2-propanol: Methanol: Acetic acid $(80: 10: 10: 0.2, \mathrm{v} / \mathrm{v})$ at a flow rate of $1.0 \mathrm{mLmin}^{-1}$. The resolution between the enantiomers was found to be not less than 3.0 in optimized method. The presence of acetic acid in the mobile phase played an important role, in enhancing chromatographic efficiency and resolution between the enantiomers. The developed method was extensively validated and proved to be robust.

The calibration curve for enantiomers showed excellent linearity over the concentration range of $5 \mu \mathrm{gmL}^{-1}$ to $50 \mu \mathrm{gmL}^{-1}$. The limit of detection and limit of quantification for enantiomers were 0.05 and $0.16 \mu \mathrm{gmL}^{-1}$, respectively. The proposed method was found to be suitable and accurate for quantitative determination of enantiomers in bulk drug substance.
\end{abstract}

Keywords: Metolazone; Method development; Enantiomeric separation; Amylose based stationary phase; Validation.

\section{INTRODUCTION}

Metolazone (fig-1) is a quinazoline a derivative of the similar diuretics quinethazone. Chemically it is 7-chloro-2-methyl-3-(2-methyl phenyl)-4oxo-1, 2-dihydroquinazoline-6-sulfonamide. Metolazone is an oral diuretic drug, commonly classified with the thiazide diuretics. It is primarily used to treat congestive heart failure and high blood pressure. Metolazone is often used together with loop diuretics such as furosemide or bumetanide. Metolazone indirectly decreases the amount of water reabsorbed in to the bloodstream by the kidney, so that blood volume decrease and urine volume increases. The action of metolazone results from interference with the renal tubular mechanism of electrolyte reabsorption. Metolazone acts primarily to inhibit sodium reabsorption at the cortical diluting site and to a lesser extent in the proximal convoluted tubule. Sodium and chloride ions are excreted in approximately equivalent amounts. Metolazone has been developed and marketed as racemate. Metolazone standard brand name in U.S. Zaroxolyn and in Canada Mykrox.<smiles>Cc1ccccc1N1C(=O)c2cc(S(N)(=O)=O)c(Cl)cc2NC1C</smiles>

Fig 1. Chemical Structure of Metolazone.

Many chiral pharmaceuticals drugs are still sold as racemate. Recent global advance in new regulatory guidelines for racemate or 'pure' pharmaceutical products necessitate development of rapid, sensitive and reproducible methods for quality control of optical antipodes present in drug substance. Determination of enantiomeric purity, or enantiomeric excess (ee), is of special importance in the control of the purity of chiral synthetic materials and chiral pharmaceuticals.

Separation of enantiomers has become very important in analytical chemistry, especially in the pharmaceutical and biological fields, because some stereoisomer of racemic drugs have quite different pharmacokinetics and different pharmacological or toxicological effects ${ }^{1}$.

The development of stereoselective HPLC method for the determination of enantiomeric drugs has received considerable attention in recent years because of its importance in analysis of quality control of pharmaceutical formulations. We developed HPLC method for the separation and quantitation of metolazone enantiomers in its pharmaceutical formulations. The objective of this study was to separate the enantiomers of metolazone. Chiral separation techniques mainly include high performance liquid chromatography (HPLC), gas chromatography
(GC), and capillary electrophoresis (CE). Chiral HPLC has been recognized as a useful methodology for the separation of chiral drugs than other techniques.

A through literature survey revealed that a few HPLC methods were reported for determination of metolazone in human urine ${ }^{2}$. Also in literature, some of HPLC methods were cited for the determination of metolazone in human plasma ${ }^{3,4}$, and pharmaceutical dosage form ${ }^{5}$.

Polysaccharide-based stationary phases are quite popular with wide recognition for direct resolution of enantiomers. To the best of our knowledge, there was no validated chiral LC method for determination of the enantiomeric purity of metolazone in bulk drugs. The objective of this work is to develop a simple, rapid and validated method for separation and validation of metolazone enantiomers on amylose based stationary phase Chiralpak AD-H column. Continuation of our research work on chiral separation ${ }^{6-11}$ in further research we developed a novel, simple and robust chiral HPLC method for metolazone . The developed method was validated with respective linearity, precision, LOD, LOQ and robustness.

\section{EXPERIMENTAL}

\section{Chemicals}

Sample of metolazone and tablet Diurem $(5 \mathrm{mg})$ were obtained from Cipla pharmaceutical limited (Mumbai, India). HPLC grade hexane and acetic acid were purchased from Qualigens Fine chemicals (Mumbai, India). The HPLC grade 2-propanol and methanol were purchased from Merck Ltd (Mumbai, India).

Equipment

HPLC system used was an Agilent Technology (1100 series, Germany), system equipped with auto sampler, quaternary pump, degasser and a UV Detector. The out put signal was monitored and processed using Agilent Chemstation software.

\section{Standard Preparation}

The stock solution of racemate $\left(5.0 \mathrm{mg} \mathrm{mL}^{-1}\right)$ was prepared by dissolving appropriate amount of substance in methanol. For quantification of enantiomers a solution of $0.5 \mathrm{mg} \mathrm{mL}^{-1}$ concentration was used.

Chromatographic condition

The chromatographic column used was 250 x $4.6 \mathrm{~mm}$ Chiralpak AD-H (Daicel Chemical Industries, Ltd., Tokyo, Japan) packed with $5 \mathrm{~mm}$ particles. The mobile phase was hexane: 2-propanol :methanol: acetic acid $(80: 10: 10: 0.2, \mathrm{v} / \mathrm{v})$. The flow rate of the mobile phase was $1.0 \mathrm{mLmin}^{-1}$. The column temperature was maintained at $30^{\circ} \mathrm{C}$ and the eluent was monitored at a wavelength of $254 \mathrm{~nm}$. The injection volume was $10 \mu \mathrm{L}$. cellulose based chiral stationary phase Chiralcel OD-H and Chiralcel OJ-H (Diacel Chemical Industries, Ltd., Tokyo, Japan) were employed during the method development.

Method Validation

The method was validated according to ICH guidelines for validation of analytical procedures ${ }^{12}$

System suitability

The System suitability was determined by injecting racemate metolazone. 
Since the enantiomers form a critical band pair in the chromatogram, the qualification criteria was resolution between two enantiomers, shown to be not less than 3.0 and tailing factor should not exceed 1.5. The separation factor (a) was calculated as the ratio of retention factors, $\mathrm{a}=\mathrm{k}_{2} / \mathrm{k}_{1}$ The resolution factor $\left(R_{s}\right)$ was calculated as $R_{s}=2\left(t_{2}-t_{1}\right) /\left(w_{1}+w_{2}\right)$ where $t_{1}, t_{2}$ refer to the retention time of the first and second enantiomers; $\mathrm{w}_{1}$ and $\mathrm{w}_{2}$ are the peak width for the first and second eluting enantiomers, respectively. System suitability parameters shown in (Table-I).

Table I. System suitability report.

\begin{tabular}{|c|c|c|c|c|c|}
\hline Enantiomers & Rt (min) & $\alpha$ & Rs & N & T \\
\hline (1)-enantiomer & 3.63 & 1.23 & 3.2 & 9563 & 1.1 \\
\hline (2)-enantiomer & 4.53 & - & - & 9187 & 1.2 \\
\hline
\end{tabular}

$\mathrm{n}=3, \mathrm{R}$ - retention time, a - enantioselectivity, Rs - USP resolution, $\mathrm{N}$ -number of theoretical plates (USP tangent method) and T - USP tailing factor.

\section{Precision}

Method reproducibility was determined by measuring repeatability and intermediate precision (between-day precision). Precision was evaluated by assaying the sample at the same concentration and during the same day. Six sample solution of $250 \mu \mathrm{gmL}^{-1}$ were prepared and assayed. The intermediate precision was also evaluated over three days by performing six successive injections each day.

Accuracy/Recovery

The recovery experiments were performed by adding known amounts of the drug in the placebo. The recovery was performed at five levels, $50 \%$, $90 \%, 100 \%, 120 \%$ and $150 \%$ of the label claim of the tablet $(5 \mathrm{mg})$. Placebo equivalent to one tablet was transferred into a $100-\mathrm{mL}$ volumetric flask, and the amounts of metolazone at $50 \%, 90 \%, 100 \%, 120 \%$ and $150 \%$ of the label claim of the tablet were added to it. The recovery samples were prepared as per the procedure mentioned. Three samples were prepared for each recovery level. The recovery values for metolazone enatiomers in pharmaceutical dosage form ranged from $98.80 \%$ to $101.12 \%$ (Table- II).

Table II. Recovery result of metolazone enantiomers.

\begin{tabular}{|c|c|c|c|c|c|}
\hline $\begin{array}{c}\text { Level of } \\
\text { addition }\end{array}$ & $\begin{array}{c}\text { Amount } \\
\text { added } \\
(\mathrm{mg})\end{array}$ & $\begin{array}{c}\text { Amount } \\
\text { found (m) } \\
\text { enentiomer1 }\end{array}$ & $\begin{array}{c}\% \\
\text { Recovery } \\
\text { enentiomer1 }\end{array}$ & $\begin{array}{c}\text { Amount } \\
\text { found (m) } \\
\text { enentiomer2 }\end{array}$ & $\begin{array}{c}\text { \% Recovery } \\
\text { enentiomer2 }\end{array}$ \\
\hline $50 \%$ & 2.5 & 2.48 & 99.21 & 2.47 & 98.80 \\
\hline $90 \%$ & 4.5 & 4.51 & 100.22 & 4.49 & 99.80 \\
\hline $100 \%$ & 5.0 & 5.05 & 101.12 & 5.02 & 100.4 \\
\hline $110 \%$ & 5.5 & 5.45 & 99.09 & 5.48 & 99.63 \\
\hline $150 \%$ & 7.5 & 7.41 & 98.88 & 7.47 & 99.60 \\
\hline
\end{tabular}

\section{Solution stability}

The solution stability and mobile phase stability experimental data confirms that sample solutions and mobile phase used during analysis was stable up to $48 \mathrm{hrs}$.

Linearity of enantiomers

Linearity was assessed by preparing six calibration sample solutions of metolazone covering from detection to $200 \%$ in mobile phase from racemate stock solution. The regression curve was obtained by plotting peak area versus concentration, using the least squares method.

Limit of detection and Limit of quantification of (R)-enantiomer (LOD \& LOQ)

Limit of detection and limit of quantification of enantiomers were achieved by injecting a series of dilute solutions of enantiomers

Determination of metolazone enantiomers in Diurem tablet

Weigh 20 tablets accurately and crushed in a small mortar. Transfer weighed quantity powder equivalent to $5 \mathrm{mg}$ metolazone in to a volumetric flask and extract methanol using magnetic stirrer. Determine metolazone concentration by taking appropriate amount in $50 \mathrm{~mL}$ volumetric and make up the volume with mobile phase.

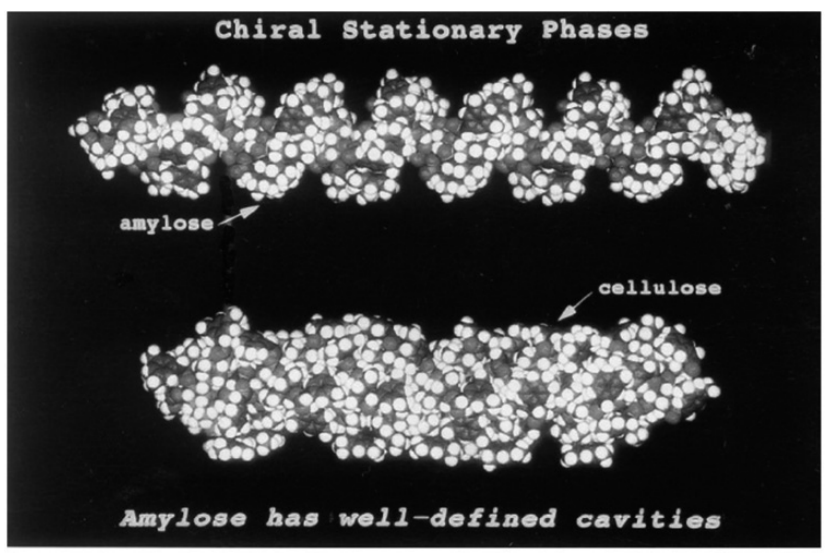

Fig.2. Chiral stationary phase (Amylose and Cellulose).

\section{RESULTS AND DISCUSSION}

\section{Optimization of chromatographic conditions}

To develop the suitable chiral HPLC method for the separation of the enantiomers of metolazone, different mobile phases and stationary phases were employed. For this, different chiral columns were used namely Chiralcel OJ-H, Chiralcel OD-H and Chiralpak AD-H. The enantiomeric separation for metolazone was not achieved by using either Chiralcel OJ-H and OD-H using mobile phase (Hexane:2-propanol:Methanol, 80:10:10, v/v). There was an indication of separation on Chiralpak AD-H column using the mobile phase consisting of hexane: 2-propanol (70:30, v/v) but the baseline resolution was not obtained. In further development it was observed that after adding methanol in combination with hexane and 2-propanol the base line resolution was obtained but peak shape was broad. For further improvement in peak shape and column efficiency, the peak modifier acetic acid was used. Better separation was achieved on chiralpak AD-H column (resolution between enantiomers was found to be >3.0) using the mobile phase Hexane:2-propanol:Methanol:Acetic acid $(80: 10: 10: 0.2, \mathrm{v} / \mathrm{v})$, which produces better resolution and chromatographic analysis time less than $7 \mathrm{~min}$. The retention time was observed at $3.1 \mathrm{~min}$ for enantiomer 1, at $4.5 \mathrm{~min}$ for enantiomer 2 .

The mechanism of chiral separation methods is the interaction of chiral stationary phase (CSP) with analytes to form short-lived, transient diastereomeric complexes. The complexes are formed as a result of hydrogen bonding, dipole-dipole interactions, pi bonding, electrostatic interactions, and inclusion complexation ${ }^{13,14}$. As discussed earlier in method development, enantiomers of metolazone could not be separated on Chiralcel OD-H and Chiralcel $\mathrm{OJ}-\mathrm{H}$ (cellulose carbamate and benzoate ester derivative of cellulose). Chiral stationary phase (CSP) that gave the best resolution was Chiralpak AD-H (3,5-tris-dimethylphenylcarbamate derivative of amylose coated on silica gel). The separation of metolazone enantiomers on chiralpak $\mathrm{AD}-\mathrm{H}$ was due to the interaction between the polar group of analytes and the polar carbamate group on the CSP. The carbamate group on the CSP interacts with the $\mathrm{NH}$ group of analytes through hydrogen bonding, the oxygen atom of metolazone form dipole-dipole interaction between CSP. The pi-pi interaction occurred between phenylcarbamate and metolazone aromatic ring, steric fit which are stabilized by insertion of aromatic portion of metolazone in to chiral grooves (asymmetric centers). Amylose forms a helical structure and posses more defined grooves (asymmetric centers) making it different than cellulose derivatives (Fig.3). These polysaccharides contain a large number of chiraly active sites and thus a relative high probability of interaction with the solute leading to separation of two enantiomers. Peak tailing may results from silanol effect, small amount of acetic acid modifier in the mobile phase to reduce peak tailing by masking the residual silanol group of the chiral stationary phase. The main advantages of developed method are the method is useful for preparative HPLC separation and routine analysis in quality control labs due to short run time and better resolution

Effect of flow rate

The flow rate of the mobile phase was $1.0 \mathrm{~mL} \mathrm{~min}^{-1}$. To study the effect of flow rate on resolution of enantiomers, it was changed 0.2 units from 0.8 to $1.2 \mathrm{~mL} \mathrm{~min}^{-1}$ while the other mobile phase components were held constant, as stated in chromatographic condition section. (Table-III)

Effect of organic modifier

The type and concentration of organic modifiers was found to influence the 
retention and resolution of metolazone enantiomers. The effect of methanol as organic modifier on resolution of metolazone enantiomers was investigated. It is clear from data that the selectivity and resolution of metolazone enantiomers is optimum with combination of methanol. (Table-III)

Effect of concentration of Acetic acid

To minimize peak tailing acetic acid was added to mobile phase on increasing the acetic acid concentration peak shape was sharpen and tailing was reduced. But increasing the acetic acid concentration from $0.05 \%$ to $0.4 \%$ decrease the peak intensity. As optimum selectivity $0.2 \%$ of acetic acid was chosen. (Table-III).

Table. III Effects of parameters on the chiral LC method.

\begin{tabular}{|l|c|c|}
\hline Prameters & Resolution* & $\alpha$ \\
\hline Flow rate $(\mathrm{mL} / \mathrm{min})$ & & \\
\hline 0.8 & 3.6 & 1.31 \\
\hline 1.0 & 3.2 & 1.24 \\
\hline 1.2 & 2.5 & 1.20 \\
\hline Column Tempereture $\left({ }^{\circ} \mathrm{C}\right)$ & & \\
\hline 25 & 3.7 & 1.29 \\
\hline 30 & 3.2 & 1.22 \\
\hline 35 & 2.7 & 1.19 \\
\hline Methanol \% in mobile phase & & \\
\hline 8 & 2.8 & 1.21 \\
\hline 10 & 3.1 & 1.23 \\
\hline 12 & 3.3 & 1.22 \\
\hline Acetic acid \% in mobile phase & & \\
\hline 0.05 & 2.1 & 1.11 \\
\hline 0.1 & 2.7 & 1.17 \\
\hline 0.2 & 3.2 & 1.22 \\
\hline 0.3 & 3.1 & 1.23 \\
\hline 0.4 & 2.9 & 1.19 \\
\hline
\end{tabular}

\section{Effect of column temperature}

The effect of column temperature on resolution and retention of metolazone enantiomer was studied in the range of $\left(25-35^{\circ} \mathrm{C}\right)$ on chiralpakAD-H column. On the increasing temperature, retentions as well as resolutions were decreased (Table-III). Under thermodynamically equilibrium conditions free energy accompanying the separation of enantiomers related to retention factor Thus, a mobile phase containing hexane:2-propanol: Methanol: Acetic acid $(80: 10: 10: 0.2 \mathrm{v} / \mathrm{v})$ was chosen for the separation of metolazone enantiomers on Chiralpak AD-H column maintained at $30^{\circ} \mathrm{C}$.

A representative chromatogram of metolazone enantiomers is shown in (Fig.3.), showing an excellent resolution $\left(\mathrm{R}_{\mathrm{s}}=3.2\right)$ between two enantiomers and symmetric peak shape with tailing 1.15 was obtained. In the optimized method, the typical retention times of metolazone enantiomer and were about 3.63 and $4.53 \mathrm{~min}$, respectively. The system suitability test results of the chiral liquid chromatographic method on Chiralpak AD-H are presented in (Table I)

The chromatographic resolution of the metolazone enantiomer peaks was used to evaluate the method robustness under modified conditions. The resolution between the enantiomers was greater than 2.5 and enantioselectivity (a) was better under all separation conditions tested, demonstrating sufficient robustness. As flow rate of mobile phase, column temperature and methanol is increased the resolution decreased.

Validation results of the method

In the precision study, the percentage relative standard deviation (R.S.D.) was less than $0.6 \%$ and $0.8 \%$ for the retention times of the enantiomers respectively. Peak area (R.S.D.) $0.8 \%$ and $1.3 \%$ for enantiomers respectively. (Table III). In the intermediate precision study, the results showed that R.S.D. values were in the same order of magnitude than those obtained for repeatability.

The limit of detection (LOD) and limit of quantification (LOQ) concentration were estimated to be 0.05 and $0.16 \mathrm{mg} \mathrm{mL}^{-1}$ for enantiomers, when signal-to-noise ratio of 3 and 10 were used as the criteria. The method precision for enantiomers at limit of quantification was less than 2.1.\% R.S.D.

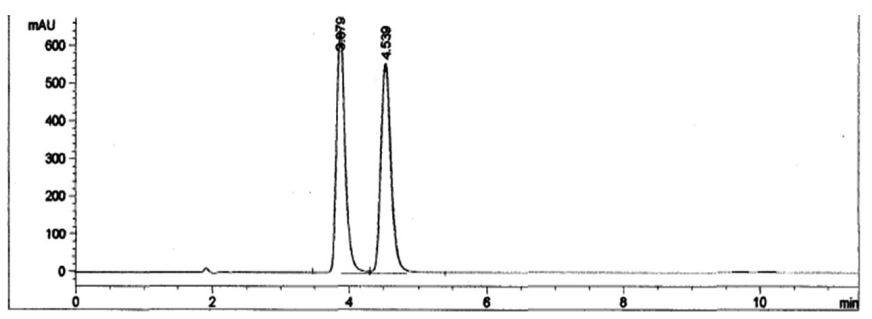

Fig. 3. Enantiomeric resolution of Metolazone enantiomers.

The described method was linear in the range of $5 \mathrm{mg} \mathrm{mL}^{-1}$ to $50.0 \mathrm{mg}$ $\mathrm{mL}^{-1}$ for enantiomer 1 and enantiomer 2 respectively. The calibration curve was drawn by plotting the peak area of enantiomer 1 and enantiomer 2 verses its corresponding concentration with correlation coefficient of 0.995 and 0997 respectively. The representative linear equations of the calibration curves for enantiomer 1 were $\mathrm{Y}=3.5356 \mathrm{x}+2.3768$ and $\mathrm{Y}=3.7577 \mathrm{x}-1.9676$ enantiomer 2 respectively.

The \% R.S.D. of metolazone content during solution stability and mobile phase stability experiments was within $1.5 \%$. Hence metolazone sample solution and mobile phase were stable for at least $48 \mathrm{hrs}$.

\section{CONCLUSION}

A simple, rapid and accurate chiral HPLC method has been developed and validated for the enantiomeric separation of metolazone. Chiralpak AD-H (amylose-based chiral stationary phase) was found to be selective for the enantiomers of the drug. Baseline separation with resolution greater than 3.8 is achieved between the two enantiomers within 7 minutes. The effect of organic modifiers and temperature on resolution and retention of enantiomers have been evaluated. The method was completely validated with respect to precision, linearity, LOD, LOQ and robustness as per ICH guidelines.

\section{ACKNOWLEDGEMENTS}

The authors are thankful to The Head, Department of Chemical Technology, Dr. Babasaheb Ambedkar Marathwada University, Aurangabad, 431004 - (M.S.), India for providing laboratory facility. We are also thankful to Cipla pharmaceutical Ltd. (Mumbai, India) for providing sample of metolazone for the research work.

\section{REFERENCES}

1. C. J. Sahajwalla, New Drug Development, Marcel Dekker Inc., NewYork, 141,421, (2004)

2. R. Schoonees, N. Y. State, J. Med. 71, 566, (1971).

3. Y. Kim, S. Kim, M. Kim, W. Lee, Rapid Comm. Mass Spectrometry. 18, 917, (2004).

4. D. Farthing, I. Fakhry, T.W. D.A Gehr, J. Chromatogr B. 534, 228, (1990).

5. R. Dubey, V.K. Bhusari, S.R. Dhaneshwar, Sci.Pharma.79, 545, (2011).

6. R.D.Yeole, A.S.Jadhav, K.R.Patil, V. P. Rane, M.L. Kubal, S.Singh, M.V. Patel, H.F. Khorakiwala, J. Chromatography A. 1108, 38, (2006).

7. V. P. Rane, D. B. Shinde, Chromatographia, 66, 583, (2007).

8. V. P. Rane, D. B. Shinde, J. Chromatogr Sci. 46, 1, (2008).

9. V. P. Rane, J.N. Sangshetti, K.R. Patil, D. B. Shinde, Chromatographia. 67,777, (2008).

10. V.P. Rane, K.R. Patil, D. B. Shinde, Die Pharmazie. 64, 8,495, (2009).

11. K. R. Patil, V. P. Rane, J. N. Sangshetti, R. D. Yeole, D. B. Shinde, J. Chil. Chem. Soc. 2, 56, (2011).

12. ICH draft Guidelines on Validation of Analytical Procedures; Definitions andTerminology, Federal Register, 60, IFPMA, Switzerland, (ICH$\mathrm{Q} 2 \mathrm{~B} / 1-8 / 96)$.

13. Y. Okamoto, A practical approach to chiral separation by LC. J. Chromatogr. A. 666, 403, (1994).

14. Y. Okamoto, Polysaccharide derivatives for chromatographic separation of enantiomer. Angew. Chem. Int.Ed. 37, 1020, (1998). 